\title{
Correction to: Norwegian reference values for the Short-Form Health Survey 36: development over time
}

\author{
Ellisiv L. Jacobsen ${ }^{1}$ Asta Bye ${ }^{2,3} \cdot$ Nina Aass $^{4,5} \cdot$ Sophie D. Fossåa, ${ }^{5,6}$ Kjersti S. Grotmol ${ }^{2} \cdot$ Stein Kaasa ${ }^{5,7}$. \\ Jon Håvard Loge ${ }^{2,4} \cdot$ Torbjørn Moum $^{8} \cdot$ Marianne J. Hjermstad $^{2,7}$
}

Published online: 21 November 2017

(c) The Author(s) 2017. This article is an open access publication

\section{Correction to: Qual Life Res \\ https://doi.org/10.1007/s11136-017-1684-4}

In the original publication of the article, the right number of participants included in the analysis should be 2107 and not 2118 as written in the paper. The flow-chart and corrected SF-36 scores for the 2015 data set for this article should have appeared as follows: Fig. 1 and Table 3. These changes did not influence the results. The authors would like to apologize for any inconvenience caused.

Also this article was originally published electronically on the publisher's internet portal (currently SpringerLink) on 14th August 2017 without open access. With the author(s)' decision to opt for Open Choice the copyright of the article changed on 20th of November to (C) The Author(s) 2017 and the article is forthwith distributed under the terms of the Creative Commons Attribution 4.0 International License (http:// creativecommons.org/licenses/by/4.0/), which permits use, duplication, adaptation, distribution and reproduction in any medium or format, as long as you give appropriate credit to the original author(s) and the source, provide a link to the Creative Commons license and indicate if changes were made. The original article was corrected.

The online version of the original article can be found under doi:https://doi.org/10.1007/s11136-017-1684-4.

Ellisiv L. Jacobsen

Ellisiv.jacobsen@hioa.no

1 Department of Physiotherapy, Faculty of Health Sciences, Oslo and Akershus University College of Applied Sciences, Oslo, Norway

2 Regional Advisory Unit for Palliative Care, Department of Oncology, Oslo University Hospital, Oslo, Norway

3 Department of Nursing and Health Promotion, Faculty of Health Sciences, Oslo and Akershus University College of Applied Sciences, Oslo, Norway

4 Faculty of Medicine, University of Oslo, Oslo, Norway
5 Department of Oncology, Oslo University Hospital, Oslo, Norway

$6 \quad$ National Advisory Unit on Late Effects After Cancer Treatment and Department for Clinical Service, Oslo University Hospital, Oslo, Norway

7 European Palliative Care Research Centre (PRC), Department of Cancer Research and Molecular Medicine, Faculty of Medicine, Norwegian University of Science and Technology (NTNU), Trondheim, Norway

8 Department of Behavioural Sciences in Medicine, Faculty of Medicine, University of Oslo, Oslo, Norway 
Fig. 1 Flowchart of subject inclusion in the three survey

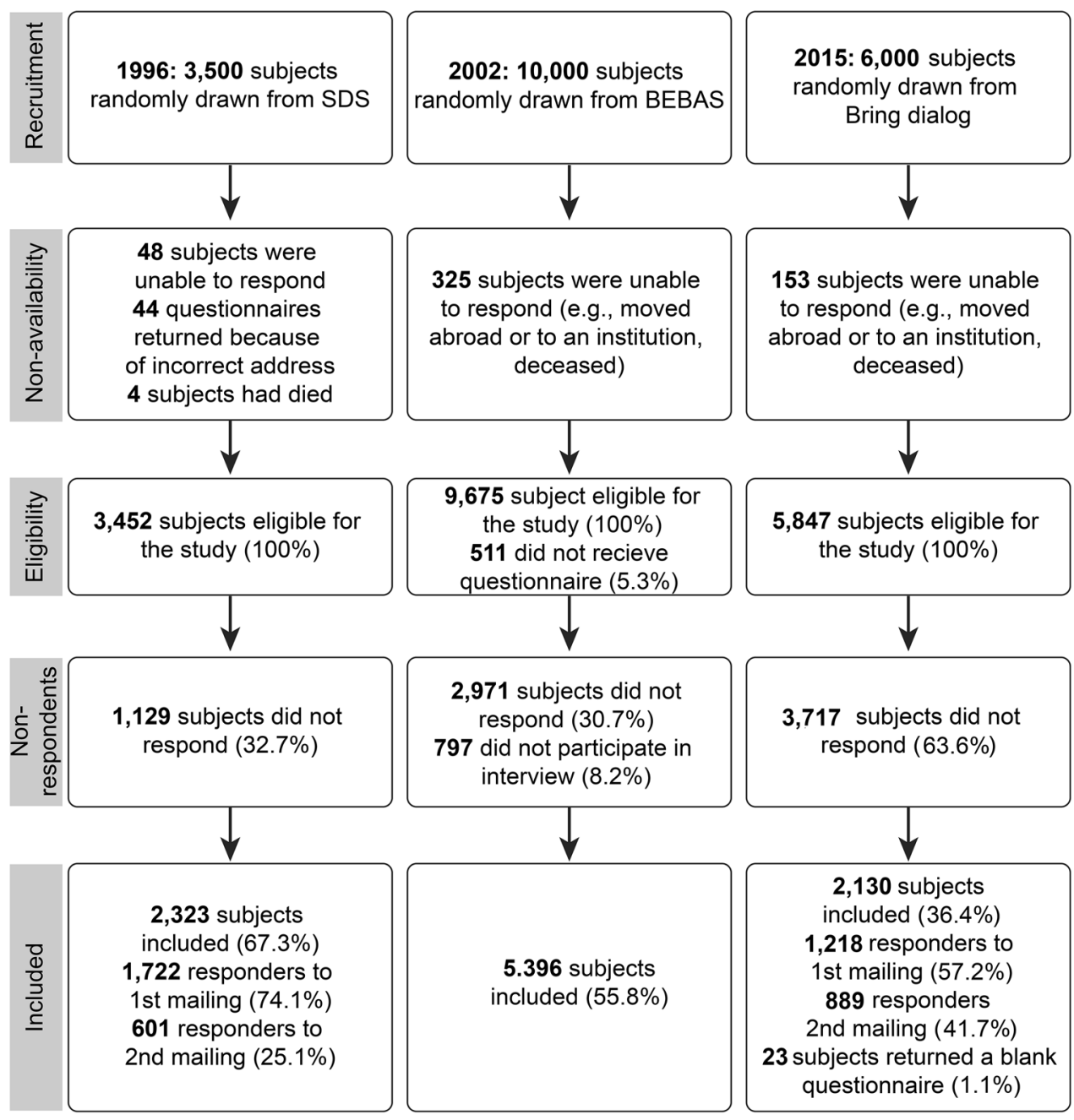




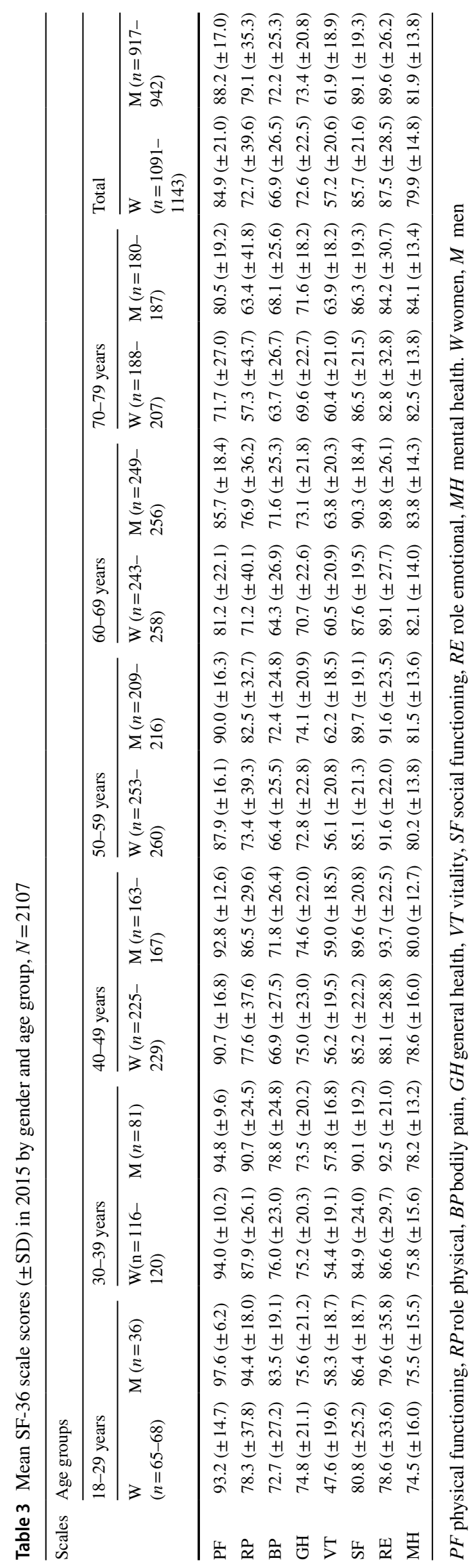

